06

\title{
Фазово-модулированный двухкомпонентный импульс в диспергирующей среде
}

\author{
(C) Г.Т. Адамашвили \\ Грузинский технический университет, \\ 0160, Тбилиси, Грузия \\ e-mail: guram_adamashvili@ymail.com
}

Поступила в редакцию 28.03.2019 г.

В окончательной редакции 18.04.2019 г.

Принята к публикации 20.06.2019 г.

\begin{abstract}
Построена теория оптического двухкомпонентного импульса самоиндуцированной прозрачности в диспергирующей среде. Используя обобщенную версию метода пертурбативного разложения, система материальных уравнений для ансамбля двухуровневых атомов и волновое уравнение в диспергирующей среде сводятся к связанным нелинейным уравнениям Шредингера. Получено решение в форме фазово-модулированного двухкомпонентного $0 \pi$-импульса, компоненты которого осциллируют на суммарной и разностной частотах в окрестности частоты несущей волны. Приведены явные аналитические выражения для параметров и условий формирования нелинейной волны, которые зависят от дисперсионных свойств среды. В частном случае получены известные результаты.
\end{abstract}

Ключевые слова: самоиндуцированная прозрачность, импульс, дисперсия, солитон.

DOI: $10.21883 /$ OS.2019.11.48518.125-19

\section{Введение}

При нелинейном когерентном взаимодействии оптического импульса с содержащимися в среде оптическими примесными резонансными атомами или полупроводниковыми квантовыми точками (ПКТ) может иметь место эффект самоиндуцированной прозрачности (СИП), когда длительность импульса значительно короче времен поперечной и продольной релаксаций резонансных атомов. В этих условиях могут формироваться резонансные нелинейные уединенные волны СИП с неизменным профилем [1].

Важным параметром СИП является площадь огибающей импульса $\Theta$, которая характеризует интенсивность взаимодействия света с веществом и определяет вид импульса СИП. Согласно теореме площадей МакКолла и Хана, когда площадь импульса превышает $\pi$, образуется оптический $2 \pi$-импульс (гиперболический секанс), а для импульса малой интенсивности, когда $\Theta<<1$, может формироваться резонансный оптический $0 \pi$-импульс как в поглощающей, так и в инверснозаселенной средах [2-11]. Несмотря на то что $2 \pi-$ и $0 \pi$-импульсы являются совершенно разными нелинейными резонансными волнами с абсолютно различными характеристиками и условиями формирования, эти импульсы обладают и общим свойством, а именно оба эти импульса являются скалярными однокомпонентными нелинейными волнами. Свойства резонансных однокомпонентных нелинейных волн СИП в различных средах и разнообразных физических ситуациях на протяжении длительного времени исследовались достаточно подробно [12-14].
Однако скалярные однокомпонентные нелинейные импульсы не являются единственно возможными нелинейными импульсами СИП. Недавно было показано, что наряду со скалярными однокомпонентными нелинейными волнами также может формироваться и двухкомпонентный импульс СИП $[15,16]$. Эта нелинейная волна представляет собой два связанных волновых пакета, которые распространяются с одинаковыми скоростями и осциллируют на суммарной и разностной частотах в окрестности частоты несущей волны $\omega$. Ввиду того, что составляющие нелинейный импульс волновые пакеты имеют идентичные поляризации, эту двухкомпонентную (векторную) волну СИП можно рассмотреть как фазовомодулированный нелинейный импульс с нулевой площадью огибающей импульса (векторный 0л-импульс). В результате профили скалярного и векторного $0 \pi$ импульсов существенно отличаются друг от друга. Как и однокомпонентные $2 \pi$ - и $0 \pi$-импульсы, двухкомпонентный векторный $0 \pi$-импульс может распространятся через резонансно-поглощающую среду без существенных энергетических потерь, т.е. также имеет свойство просветления резонансной среды $[15,16]$.

В терминах теории солитонов однокомпонентные $2 \pi$ и $0 \pi$-импульсы СИП являются скалярными солитоном и бризером, а резонансный двухкомпонентный $0 \pi$-импульс является векторным солитоном, так как является решением связанных нелинейных уравнений Шредингера, хотя и имеет специфическую особенность - осциллирует на суммарной и разностной частотах.

При теоретическом описании явления СИП следует исходить из системы уравнений Блоха-Максвелла для медленно меняющихся огибающих напряженности элек- 
трического поля импульса и поляризации оптических атомов (ПКТ), когда длительность импульса $T>>1 / \omega$. При анализе этих уравнений для изучения свойств однокомпонентных скалярных $2 \pi$ - и $0 \pi$-импульсов СИП обычно ограничиваются в волновом уравнении первыми производными напряженности электрического поля волны по временной и пространственной переменным, пренебрегая соответствующими вторыми производными. Тем не менее вполне возможно получить все известные результаты СИП [1-8,17]. Учет вклада от вторых производных приводит лишь к относительно малым поправкам для параметров однокомпонентных импульсов СИП $[18,19]$.

Однако относительно недавно было показано, что учет в волновом уравнении вторых производных напряженности электрического поля волны по временной и пространственной переменным может привести и к качественно новым результатам $[15,16]$. Это становится возможным при условии более общего рассмотрения явления СИП и соответствующих уравнений, если применить обобщенную версию метода пертурбативного разложения (см. (7)). С помощью такого разложения становится возможным расширить число характеризующих волновой процесс параметров, получить связанное состояние двух волновых пакетов и как результат получить векторный $0 \pi$-импульс СИП.

Изучение свойств резонансных нелинейных волн в различных средах относится к числу основных задач теории нелинейных оптических волн. В диспергирующих средах свойства скалярных однокомпонентных солитонов и бризеров изучены достаточно подробно $[2,13,18,19]$. При этом, хотя свойства двухкомпонентных векторных 0л-импульсов СИП рассмотрены во многих системах и в различных физических ситуациях $[15,16,20-22]$ как для плоских, так и поверхностных и волноводных мод, их свойства в диспергирующих средах не были изучены. Цель настоящей работы исследовать именно этот вопрос.

\section{Вывод уравнений}

Рассмотрим процесс формирования оптического векторного 0л-импульса СИП в изотропной диспергирующей среде второго порядка. В общем случае в кристаллах и наноструктурах эффекты, связанные с дисперсией, исследуют с помощью тензора диэлектрической проницаемости $\varepsilon_{i j}(z, t)$, который зависит от времени $t$ и пространственной координаты $z$, где $i, j=x, y, z$. Но в изотропных средах тензор диэлектрической проницаемости упрощается и трансформируется к виду $\varepsilon_{i j}(z, t)=\varepsilon(z, t) \delta_{i j}$, где $\varepsilon(z, t)$ является скалярной функцией, $\delta_{i j}$ - символ Кронекера.

Рассматривается модель, когда в диспергирующую матрицу внедряется малая концентрация $n_{0}$ примесных оптических атомов или ПКТ. Предположим, что оптическая линейно-поляризованная вдоль оси $x$ волна (с длительностью $T$, частотой $\omega$ и волновым вектором $\mathbf{k}$ несущей волны) расспространяется вдоль положительного направления оси $z$.

Волновое уравнение для $x$-компоненты напряженности электрического поля импульса $\mathbf{E}(E, 0,0)$ в диспергирующей среде имеет вид [23]

$$
\begin{aligned}
-C^{2} \frac{\partial^{2} E}{\partial z^{2}} & +\frac{\partial^{2}}{\partial t^{2}} \int \varepsilon\left(z_{1}, t_{1}\right) \\
& \times E\left(z-z_{1}, t-t_{1}\right) d z_{1} d t_{1}+4 \pi \frac{\partial^{2} P}{\partial t^{2}}=0,
\end{aligned}
$$

где $C-$ скорость света в вакууме. $P=n_{0} \mu s_{1}$ является $x$-компонентой вектора поляризации системы двухуровневых атомов или ПКТ, $\mu$ - электродипольный момент для соответствующих квантовых переходов оптических атомов или ПКТ, $s_{i}(t)=<\hat{\sigma}_{i}(t)>-$ средние значения операторов Паули $\hat{\sigma}_{i}(i=1,2,3)$.

Зависимость поляризации $P$ от напряженности электрического поля импульса определяется из уравнений Блоха $[1,2]$ :

$$
\begin{gathered}
\frac{\partial s_{1}}{\partial t}=-\omega_{0} s_{2}, \\
\frac{\partial s_{2}}{\partial t}=\omega_{0} s_{1}+\kappa_{0} E s_{3}, \\
\frac{\partial s_{3}}{\partial t}=-\kappa_{0} E s_{2},
\end{gathered}
$$

где $\kappa_{0}=\frac{2 \mu}{\hbar}, \hbar-$ постоянная Планка, $\omega_{0}-$ резонансная частота возбуждения примесных двухуровневых атомов или ПКТ.

Система уравнений Максвелла-Блоха (1) и (2) представляет собой систему уравнений СИП в диспергирующей среде, содержащей примесные оптические двухуровневые атомы. Учитывая, что длительность импульса $T>>\omega^{-1}$, эти уравнения можно упростить, используя метод медленно меняющейся огибающей. Для этой цели представим $x$-компоненты напряженности электрического поля импульса $E$ и поляризации $P$ в следующей форме $[2,3,12]$ :

$$
E=\sum_{l= \pm 1} \hat{E}_{l} Z_{l}, \quad P=n_{0} \mu \sum_{l} d_{-l} Z_{l},
$$

где $\hat{E}_{l}$ и $d_{l}$ - медленно меняющиеся комплексные амплитуды электрического поля волны и поляризации оптически активных примесных двухуровневых атомов или ПКТ, $Z_{l}=e^{i l(k z-\omega t)}$. Учитывая, что функция $E$ вещественна, получаем условие $\hat{E}_{l}=\hat{E}_{-l}^{*}$. Комплексные огибающие $\hat{E}_{l}$ и $d_{l}$ меняются достаточно медленно по пространственной координате и времени по сравнению с быстро осциллирующей части $Z_{l}$, т.е. имеют место неравенства

$$
\begin{gathered}
\left|\frac{\partial \hat{E}_{l}}{\partial t}\right| \ll \omega\left|\hat{E}_{l}\right|, \quad\left|\frac{\partial \hat{E}_{l}}{\partial z}\right| \ll k\left|\hat{E}_{l}\right|, \\
\left|\frac{\partial d_{l}}{\partial t}\right| \ll \omega\left|d_{l}\right|, \quad\left|\frac{\partial d_{l}}{\partial z}\right|<<k\left|d_{l}\right| .
\end{gathered}
$$


Подставляя уравнение (3) в (1), получаем закон дисперсии для импульса в диспергирующей среде:

$$
C^{2} k^{2}=\omega^{2} \kappa
$$

и нелинейное волновое уравнение для огибающей функции $\hat{E}_{l}$ в форме

$$
\begin{gathered}
\sum_{l= \pm 1} Z_{l}\left\{\left[i G_{1, l} \frac{\partial \hat{E}_{l}}{\partial z}+i g_{3} \frac{\partial \hat{E}_{l}}{\partial t}+G_{2} \frac{\partial^{2} \hat{E}_{l}}{\partial z^{2}}\right.\right. \\
\left.-g_{5} \frac{\partial^{2} \hat{E}_{l}}{\partial t^{2}}-g_{6} \frac{\partial^{2} \hat{E}_{l}}{\partial z \partial t}\right]+4 \pi \frac{\partial^{2} P}{\partial t^{2}}=0,
\end{gathered}
$$

где

$$
\begin{gathered}
G_{1, l}=\omega^{2} a-2 l k C^{2}, \\
G_{2}=\omega^{2} c-C^{2} . \\
g_{3}=-\left(\omega^{2} b+2 l \omega \kappa\right), \\
g_{5}=-\left(\omega^{2} d+2 l \omega b+\kappa\right), \\
g_{6}=2 l \omega a+\omega^{2} t . \\
\kappa=\int \varepsilon\left(z_{1}, t_{1}\right) e^{-i l\left(k z_{1}-\omega t_{1}\right)} d z_{1} d t_{1}, \\
a=-i \int \varepsilon\left(z_{1}, t_{1}\right) z_{1} e^{-i l\left(k z_{1}-\omega t_{1}\right)} d z_{1} d t_{1}, \\
b=i \int \varepsilon\left(z_{1}, t_{1}\right) t_{1} e^{-i l\left(k z_{1}-\omega t_{1}\right)} d z_{1} d t_{1}, \\
c=-\int \varepsilon\left(z_{1}, t_{1}\right) \frac{z_{1}{ }^{2}}{2} e^{-i l\left(k z_{1}-\omega t_{1}\right)} d z_{1} d t_{1}, \\
d=-\int \varepsilon\left(z_{1}, t_{1}\right) \frac{t_{1}{ }^{2}}{2} e^{-i l\left(k z_{1}-\omega t_{1}\right)} d z_{1} d t_{1}, \\
t=\int \varepsilon\left(z_{1}, t_{1}\right) t_{1} z_{1} e^{-i l\left(k z_{1}-\omega t_{1}\right)} d z_{1} d t_{1} .
\end{gathered}
$$

Коэффициенты (6) содержат всю информацию о дисперсионных свойствах материала [24,25].

\section{Решение уравнений}

Для анализа системы уравнений (2) и (5) можем использовать пертурбативный метод редукции (ПМР), предложенный в работе [26]. С помощью этого метода можно трансформировать систему этих уравнений к нелинейному уравнению Шредингера, которое имеет решение в виде однокомпонентного скалярного солитона (бризера) [3-5], в том числе и в диспергирующей среде $[18,19]$.

Для того чтобы исследовать двухкомпонентный векторный $0 \pi$-импульс СИП, необходимо обобщить ПМР [26] и представить комплексную функцию $\hat{E}_{l}(z, t)$ в следующем виде $[15,16,20-22]$ :

$$
\hat{E}_{l}(z, t)=\sum_{\alpha=1} \varepsilon^{\alpha} \hat{E}_{l}^{(\alpha)}=\sum_{\alpha=1}^{\infty} \sum_{n=-\infty}^{+\infty} \varepsilon^{\alpha} Y_{l, n} f_{l, n}^{(\alpha)}\left(\xi_{l, n}, \tau\right)
$$

где

$$
Y_{l, n}=e^{i n\left(Q_{l, n} z-\Omega_{l, n} t\right)}, \quad \xi_{l, n}=\varepsilon Q_{l, n}\left(z-v_{l, n} t\right),
$$

$$
\tau=\varepsilon^{2} t, \quad v_{g ; l, n}=\frac{d \Omega_{l, n}}{d Q_{l, n}}
$$

$\varepsilon$ - малый параметер.

В отличие от ПМР в разложении (7) мы рассматриваем ситуацию, когда величины $Q_{l, n}$ и $\Omega_{l, n}$ зависят от индексов $l$ и $n$, тем самым увеличивая количество параметров, характеризующих волновой процесс. В разложении (7) выделяются из функции $\hat{E}_{l}$ еще более медленно меняющиеся функции $f_{l, n}^{(\alpha)}$. Следовательно, предполагается выполнение для величин $\Omega_{l, n}, Q_{l, n}$ и $f_{l, n}^{(\alpha)}$ следующих неравенств для любых значений индексов $l$ и $n$ :

$$
\begin{gathered}
\omega \gg \Omega, \quad k \gg Q, \\
\left|\frac{\partial f_{l, n}^{(\alpha)}}{\partial t}\right| \ll \Omega\left|f_{l, n}^{(\alpha)}\right|, \\
\left|\frac{\partial f_{l, n}^{(\alpha)}}{\partial z}\right| \ll Q\left|f_{l, n}^{(\alpha)}\right| .
\end{gathered}
$$

Хотя параметры $\Omega_{l, n}, Q_{l, n}, \xi_{l, n}$ и $v_{l, n}$ зависят от индексов $l$ и $n$, для простоты опустим эти индекси везде, где это не приведет к путанице.

Подставляя уравнения (3) и (7) в уравнения Блоха (2), при неоднородном уширении спектральной линии получаем поляризацию ансамбля двухуровневых атомов в форме

$$
\begin{aligned}
P= & i \frac{n_{0} \mu^{2}}{\hbar} \int \frac{g(\Delta) d \Delta}{1+T^{2} \Delta^{2}} \sum_{l= \pm 1} l Z_{l}\left[\varepsilon^{1} \Theta_{l}{ }^{(1)}+\varepsilon^{2} \Theta_{l}{ }^{(2)}+\varepsilon^{3} \Theta_{l}{ }^{(3)}\right. \\
& \left.-\varepsilon^{3} \frac{\kappa_{0}^{2}}{2} \int \frac{\partial \Theta_{l}^{(1)}}{\partial t} \Theta_{-l}^{(1)} \Theta_{l}^{(1)} d t^{\prime}\right]+\mathcal{O}\left(\epsilon^{4}\right)
\end{aligned}
$$

где

$$
\Theta_{l}^{(\alpha)}(z, t)=\int_{-\infty}^{t} \hat{E}_{l}^{(\alpha)}\left(z, t^{\prime}\right) d t^{\prime}
$$

$g(\Delta)$ - нормированная функция неоднородного уширения спектральной линии для ансамбся двухуровневых оптических атомов, $\Delta=\omega_{0}-\omega$.

Подставляя уравнения (7) в (5) и учитывая явный вид для поляризации $(8)$, получим

$$
\begin{aligned}
& \sum_{l= \pm 1} \sum_{\alpha=1}^{\infty} \sum_{n=-\infty}^{+\infty} \varepsilon^{\alpha} Y_{l, n} Z_{l}\left\{\tilde{W}_{l, n}\right. \\
& \left.\quad+i \varepsilon J_{l, n} \frac{\partial}{\partial \xi}+i \varepsilon^{2} h_{l, n} \frac{\partial}{\partial \tau}+\varepsilon^{2} H_{l, n} \frac{\partial^{2}}{\partial \xi^{2}}\right\} f_{l, n}^{(\alpha)} \\
& \left.\quad=-\varepsilon^{3} i æ_{2} \sum_{l= \pm 1} l Z_{l} \int \frac{\partial \Theta_{l}^{(1)}}{\partial t} \Theta_{-l}^{(1)} \Theta_{l}^{(1)} d t^{\prime}+\mathscr{O}\left(\epsilon^{4}\right)\right\}
\end{aligned}
$$

где

$$
\tilde{W}_{l, n}=g_{3} n \Omega-G_{1, l} n Q-G_{2} Q^{2}+g_{5} \Omega^{2}-g_{6} Q \Omega+\mathfrak{x}_{1} \frac{\ln }{\Omega},
$$




$$
\begin{gathered}
J_{l, n}=g_{3} v_{g}-G_{1, l}-2 G_{2} n Q+2 n g_{5} \Omega v_{g}-g_{6} n\left(Q v_{g}+\Omega\right), \\
h_{l, n}=g_{3}+2 g_{5} n \Omega-g_{6} n Q \\
H_{l, n}=Q^{2}\left(G_{2}-g_{5} v_{g}^{2}+g_{6} v_{g}\right) \\
\mathfrak{x}_{1}=\frac{4 \pi \omega^{2} n_{0} \mu^{2}}{\hbar} \int \frac{g(\Delta) d \Delta}{1+T^{2} \Delta^{2}} \\
\mathfrak{x}_{2}=\frac{2 \mu^{2}}{\hbar^{2} \omega^{2}} \mathfrak{x}_{1}
\end{gathered}
$$

Для определения из уравнения (9) функций $f_{l, n}^{(\alpha)}$, приравняем нулю различные члены при одинаковых степенях малого параметра $\varepsilon$. В результате получаем цепочку уравнений. В первом порядка по $\varepsilon$ получаем, что из всех функциий $f_{l, n}^{(1)}$ отличны от нуля только функции $f_{ \pm 1, \pm 1}^{(1)}$ или $f_{ \pm 1, \mp 1}^{(1)}$, а соотношение между параметрами $\Omega$ и $Q$ определяется из уравнения (10) и имеет следующий вид $(l= \pm 1, n= \pm 1)$ :

$$
\begin{aligned}
& g_{3} n \Omega-G_{1, l} n Q-G_{2} Q^{2} \\
& \quad+g_{5} \Omega^{2}-g_{6} Q \Omega+\mathfrak{x}_{1} \frac{\ln }{\Omega}=0 .
\end{aligned}
$$

Подставляя уравнения (11) в (10), во втором порядка по $\varepsilon$, мы можем доказать справедливость условий $J_{ \pm 1, \pm 1}=J_{ \pm 1, \mp 1}=0$. Уравнение (9) в третьем порядке по $\varepsilon$ приводит к следующему нелинейному уравнению

$$
\begin{aligned}
& {\left[i\left(\frac{\partial u_{ \pm 1}}{\partial t}+v_{ \pm} \frac{\partial u_{ \pm 1}}{\partial z}\right)+p_{ \pm} \frac{\partial^{2} u_{ \pm 1}}{\partial z^{2}}\right.} \\
& \quad+g_{ \pm}\left|u_{ \pm 1}\right|^{2} u_{ \pm 1}+r_{ \pm}\left|u_{\mp 1}\right|^{2} u_{ \pm 1}=0
\end{aligned}
$$

где

$$
\begin{gathered}
u_{ \pm}=\varepsilon f_{+1, \pm 1}, \quad v_{ \pm}=v_{g ;+1, \pm 1}, \quad p_{ \pm}=\frac{H_{+1, \pm 1}}{h_{+1, \pm 1} Q^{2}} \\
g_{ \pm}= \pm \frac{\mathfrak{x}_{2}}{\Omega_{ \pm}^{3} h_{+1, \pm 1}} \\
r_{ \pm}= \pm \frac{\mathfrak{x}_{2}}{\Omega_{ \pm}^{2} \Omega_{\mp} h_{+1, \pm 1}}\left(\frac{\Omega_{ \pm}}{\Omega_{\mp}}-1\right) .
\end{gathered}
$$

$u_{+}$и $u_{-}-$медленно меняющиеся комплексные амплитуды огибающих волновых пакетов, осциллирующих на суммарной и разностной частотах, $\Omega_{+}=\Omega_{ \pm 1, \pm 1}, \quad \Omega_{-}=\Omega_{ \pm 1, \mp 1}, \quad Q_{+}=Q_{ \pm 1, \pm 1}$, $Q_{-}=Q_{ \pm 1, \mp 1}$. Нелинейные уравнения (12) являются связанными нелинейными уравнениями Шредингера, которые имеют двухкомпонентное солитонное решение.

Для решения уравнений (12) будем следовать работам $[15,16,20-22]$, в результате получаем решение для $x$ компоненты напряженности электрического поля волны

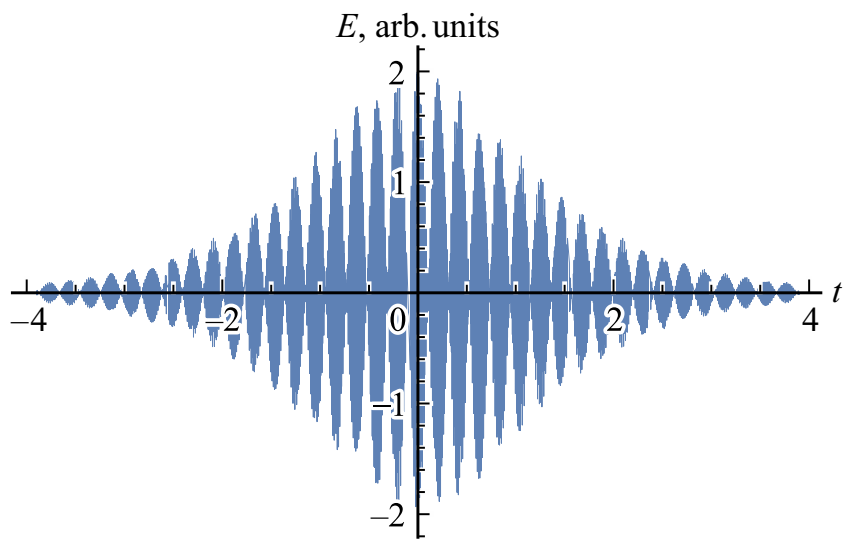

Профиль зависимости векторного 0л-импульса СИП для $x$-компоненты напряженности электрического поля импульса $E(14)$ от времени при $z=0$. Темные части рисунка представляют собой области осцилляций.

в диспергирующей среде:

$$
\begin{aligned}
& E(z, t)=\frac{\hbar}{\mu b T} \operatorname{Sech}\left(\frac{t-\frac{z}{V_{0}}}{T}\right)\left\{\left(\Omega_{+}+\omega_{+}\right)\right. \\
& \times f_{+} \sin \left[\left(k+Q_{+}+k_{+}\right) z-\left(\omega+\Omega_{+}+\omega_{+}\right) t\right] \\
& \left.-\left(\Omega_{-} \omega_{-}\right) f_{-} \sin \left[\left(k-Q_{-}+k_{-}\right) z_{-}\left(\omega-\Omega_{-}+\omega_{-}\right) t\right]\right\},
\end{aligned}
$$

где связь между параметрами волны имеет вид

$$
\begin{gathered}
f_{+}^{2}=\frac{p_{+} q_{-}-p_{-} r_{+}}{p_{-} q_{+}-p_{+} r_{-}} f_{-}^{2}, \\
\omega_{+}=\frac{p_{+}}{p_{-}} \omega_{-}+\frac{V_{0}^{2}\left(p_{-}^{2}-p_{+}^{2}\right)+v_{-}^{2} p_{+}^{2}-v_{+}^{2} p_{-}^{2}}{4 p_{+} p_{-}^{2}}, \\
T^{-2}=V_{0}^{2} \frac{v_{+} k_{+}+k_{+}^{2} p_{+}-\omega_{+}}{p_{+}}, \quad b^{2}=V_{0}^{2} \frac{f_{+}^{2} q_{+}+f_{-}^{2} r_{+}}{2 p_{+}}, \\
k_{ \pm}=\frac{V_{0}-v_{ \pm}}{2 p_{ \pm}}, \quad k_{ \pm 1}<<Q_{ \pm}, \quad \omega_{ \pm}<<\Omega_{ \pm},
\end{gathered}
$$

$V_{0}$ - скорость нелинейной волны.

На рисунке представлен профиль зависимости векторного 0л-импульса СИП для $x$-компоненты напряженности электрического поля импульса $E$ (14) от времени (в произвольных единицах) при $z=0$. При этом параметры осцилляций $\Omega_{ \pm}$на три порядка ниже частоты несущей волны $\omega$, а $\frac{\Omega_{+}}{\Omega_{-}}=1.04$.

\section{Обсуждение результатов}

Используя обобщенный метод пертурбативного разложения (7), система уравнений Блоха для ансамбля двухуровневых атомов (2) и волновое уравнение в диспергирующей среде (5) трансформируется к связанным нелинейным уравнениям Шредингера (12). Показано, что в этих условиях можно получить для $x$ компоненты напряженности электрического поля волны 
$E$ фазово-модулированный двухкомпонентный векторный 0л-импульс СИП (14), компоненты которого осциллируют на суммарной, $\omega+\Omega_{+}+\omega_{+}\left(k+Q_{+}+k_{+}\right)$, и разностной $\omega-\Omega_{-}+\omega_{-}\left(k-Q_{-}+k_{-}\right)$, частотах (волновых числах) в окрестности частоты (волнового числа) несущей волны. Параметры нелинейной волны зависят от диспергирующих свойств среды и определяются из выражениями (10), (13) и (15). Закон дисперсии и связь между величинами $\Omega_{ \pm}$и $Q_{ \pm}$определяются из выражений (4) и (11).

Суммируя полученные результаты, можно сделать заключение, что для формирования векторного 0лимпульса СИП (14) в диспергирующих средах необходимо выполнение следующих условий.

1. В волновом уравнений (5) необходимо сохранить наряду с первыми производными $\frac{\partial \hat{E}_{l}}{\partial t}$ и $\frac{\partial \hat{E}_{l}}{\partial z}$, также и вторые производные $\frac{\partial^{2} \hat{E}_{l}}{\partial z^{2}}, \frac{\partial^{2} \hat{E}_{l}}{\partial t^{2}}$ и $\frac{\partial^{2} \hat{E}_{l}}{\partial z \partial t}$ напряженности электрического поля волны по временной и пространственной переменным.

Если пренебречь в уравнении (5) вторыми производными, то в уравнении (11) члены, пропорциальные $\Omega^{2}$, $Q \Omega$ и $Q^{2}$, будут отсутствовать, уравнение $(11)$ не будет зависить от индексов $l$ и $n$, и получим, что

$$
\Omega_{+}=\Omega_{-}=\Omega, \quad Q_{+}=Q_{-}=Q, \quad r_{ \pm}=0 .
$$

Следоватеьно, связаные нелинейные уравнения Шредингера (12) расцепляются, и получаем два несвязанных с друг другом волновых пакета.

2. Необходимо наличие фазовой модуляции, т.е. величины $\hat{E}_{l}(z, t)$ должны быть комплексными функциями и должны зависить от индекса $l$. Только в этом случае можем применить обобщенный метод пертурбативного разложения (7), расширить число характеризующих волновой процесс параметров $\Omega_{ \pm}$и $Q_{ \pm}$и как результат получить решение (14).

В противном случае, при отсутствии фазовой модуляции, мы не сможем применить разложение (7) для решения уравнения (5), так как величина $\hat{E}_{l}=\hat{E}_{-l}=\hat{E}$ становится вещественной, функции $f_{l, n}^{(\alpha)}$ трансформируются к функциям $f_{n}^{(\alpha)}$, и будут выполняться условия (16).

3. Для существования решений уравнений (12) необходимо, чтобы величины $p_{+}$и $g_{+}$, а также $p_{-}$и $g_{-}$были величинами одного порядка одновременно.

Так как эти величины зависят от параметров дисперсии (6), в зависимости от характера дисперсии будут меняться и критерии существования векторного 0лимпульса СИП (16) в диспергирующей среде. При изменении параметров импульса $p_{ \pm}, g_{ \pm}, r_{ \pm}$, например при учете дисперсии, профиль векторного 0л-импульса СИП будет также меняться, но по-прежнему характерная форма импульса (14) будет сохроняться.

При отсутствии дисперсии в уравнениях (6) $a=b=c=d=t=0$, и все представленные выше выражения трансформируются к известным результатам, полученным в работе [16].
Решение (14) получено для поглощающей среды, когда до поступления в среду импульса все примесные оптические атомы находятся в основном состоянии. Однако полученый результат можно трансформировать и для инверсно-заселенной среды, если заменить знаки перед коэффициентами $\mathfrak{x}_{1}$ и $\mathfrak{x}_{2}$ в выражениях (10), (13) и (15). Это может оказаться интересным в связи с тем, что скалярные 0л-импульсы изучены в лазерах как теоретически, так и экспериментально [9-11].

Совместно с уже известными свойствами однокомпонентных $2 \pi$ - и $0 \pi$-импульсов полученные в настоящей работе результаты для двухкомпонентного векторного $0 \pi$-импульса позволяют получить более полную физическую картину теории СИП в диспергирующих средах.

\section{Финансирование работы}

Работа выполнена в рамках проекта 217064 ННФ им. Ш. Руставели.

\section{Конфликт интересов}

Авторы заявляют, что у них нет конфликта интересов.

\section{Список литературы}

[1] McCall S.L., Hahn E.L. // Phys. Rev. 1969. V. 183. P. 457.

[2] Allen L., Eberly J.H. Optical Resonance and Two-level Atoms. Wiley-Interscience. 1975. 202 р.; Аллен Л., Эберли Джс. // Оптический резонанс и двухуровневые атомы. М.: Мир. 1978. C. 222.

[3] Newell A.C. Solitons in Mathematics and Physics. Society for Industrial and Applied Mathematics, 1985. 323 p.; Ньюэлл A. // Солитоны в математике и физике.М.: Мир, 1989. $324 \mathrm{c}$.

[4] Adamashvili G.T., Manakov S.V. // Solid Stat. Commun. 1983. V. 48. P. 381.

[5] Adamashvili G.T., Knorr A. // Optics Lett. 2006. V. 31. P. 74.

[6] Адамашвили Г.Т. // Опт. и спектр. 1998. Т. 85. № 1. С. 95.

[7] Crisp M.D. // Phys. Rev. A. 1970. V. 2. P. 2172.

[8] Rothenberg J.E., Grischkowsky D., Balant A.C. // Phys. Rev. Lett. 1984. V. 53. P. 552.

[9] Arkhipov R.M., Arkhipov M.V., Babushkin I., Rosanov N.N. // Optics Lett. 2016. V. 41. P. 737.

[10] Harvey J.D., Dudley J.M., Curley P.F., Spielmann C., Krausz F. // Optics Lett. 1994. V. 19. P. 972.

[11] Arkhipov M.V., Shimko A.A., Arkhipov R.M., Babushkin I., Kalinichev A.A., Demircan A., Morgner U., Rosanov N.N. // Laser Phys. Lett. 2018. V. 15. P. 075003.

[12] Полуэктов И.А., Попов Ю.М., Ройтберг В.С. // УФН. 1974. T. 114. C. 97.

[13] Maimistov A.I., Basharov A.M., Elyutin S.O., Sklyarov Yu.M. // Phys. Rep. 1990. V. 1. P. 191.

[14] Адамашвили Г.Т. // Опт. и спектр. 2018. Т. 125. № 2. С. 269.

[15] Adamashvili G.T. // Results in Physics. 2011. V. 1. P. 26. (journal homepage:www.elsevier.com/locate/rinp.)

[16] Адамашвили Г.Т. // Опт. и спектр. 2012. Т. 113. № 1. С. 83.

[17] Адамашвили Г.Т. // Опт. и спектр. 1996. Т. 80. № 2. С. 251.

[18] Adamashvili G.T., Maradudin A.A. // Phys. Rev. E. 1997. V. 55. P. 7712. 
[19] Adamashvili G.T., Kaup D.J. // Phys. Rev. E. 2004. V. 70. P. 066616.

[20] Adamashvili G.T. // Phys. Lett. A. 2015. V. 379. P. 218.

[21] Adamashvili G.T. // Eur. Phys. J. D. 2012. V. 66. P. 101.

[22] Adamashvili G.T. // Physica B. 2012. V. 407. P. 3413

[23] Ландау Л.Д., Лифиии, Е.М. Электродинамика сплошных сред. М.: Изд-во техн. теор. лит-ры, 1957. 457 с.

[24] Виноградова М.Б., Руденко О.В., Сухоруков А.П. Теория волн. М.: Наука, 1990. 432 с.

[25] Агранович В.М., Гинзбург В.Л. Кристаллооптика с учетом пространственной дисперсии и теория экситонов. М.: Наука, 1979. 432 с.

[26] Taniuti T., Iajima N. // J. Math. Phys. 1973. V. 14. P. 1389. 